\title{
Status of the AMoRE experiment to search for Neutrinoless Double Beta Decay of Mo-100
}

\author{
Young Soo Yoon* \\ on behalf of the AMoRE Collaboration \\ Center for Underground Physics \\ Institute for Basic Science \\ E-mail: ysyeibs.re.kr
}

The AMoRE (Advanced Mo-based Rare process Experiment) is an experimental search for neutrinoless double beta decay of ${ }^{100} \mathrm{Mo}$ using enriched in ${ }^{100} \mathrm{Mo}$ and depleted in ${ }^{48} \mathrm{Ca}$ calcium molybdate $\left({ }^{48 \mathrm{depl}} \mathrm{Ca}^{100} \mathrm{MoO}_{4}\right)$ scintillating crystals at low temperature aiming to investigate the inverted hierarchy of the neutrino mass pattern. The AMoRE uses metallic magnetic calorimeter sensors to read out scintillation and phonon signals from the crystals at milli-Kelvin temperature, which provide an excellent energy resolution ( $\sim 10 \mathrm{keV} \mathrm{FWHM}$ at $2615 \mathrm{keV})$ and very efficient particle discrimination to suppress background caused by U/Th contamination of the crystal scintillators and near materials. The AMoRE experiment is intended to reach zero-background level in the region of interest, near the energy of ${ }^{100}$ Mo double beta decay $3034 \mathrm{keV}$. Monte Carlo simulations using the GEANT4 code and measurements of radioactive contamination of the ${ }^{48 \mathrm{depl}} \mathrm{Ca}^{100} \mathrm{MoO}_{4}$ crystal scintillators, detectors and shielding materials are in progress. Currently, the AMoRE pilot experiment with five ${ }^{48 \mathrm{depl}} \mathrm{Ca}^{100} \mathrm{MoO}_{4}$ detectors (total mass $\sim 1.5 \mathrm{~kg}$ ) is running at the Yang Yang underground laboratory (Korea). The first phase of the AMoRE experiment, using $\sim 5 \mathrm{~kg}$ of ${ }^{48 \mathrm{depl}} \mathrm{Ca}^{100} \mathrm{MoO}_{4}$ detectors, is scheduled to start at the end of 2017. Preparations for the first phase experiment and R\&D for the second phase experiment with $\sim 200 \mathrm{~kg}$ of molybdate crystal scintillators is ongoing.

35th International Cosmic Ray Conference ICRC2017-

10-20 July, 2017

Bexco, Busan, Korea

${ }^{*}$ Speaker. 


\section{Introduction}

Experiments with solar, atmospheric, and reactor neutrinos have provided evidences for neutrino oscillations, transitions in flight between the different flavour neutrinos, indicating nonzero neutrino masses and neutrino mixing [1]. However, their absolute mass scale and nature (Dirac or Majorana particles) are still unknown. The investigation of neutrinoless double beta decays $(0 v \beta \beta)$ is the only practical way to determine an absolute mass scale and the nature of the neutrino[2]. Neutrinoless double beta decay is expected to be very rare process $\left(\mathrm{t}_{1 / 2}>10^{25}\right.$ years) with a peak at Q-value. In order to measure such decays, measurements with very good energy resolution and very low background, i.e. zero-background level, are powerful advantages in the experimental aspects.

The Advanced Mo-based Rare process Experiment (AMoRE) [3] is an experimental search for $0 v \beta \beta$ of ${ }^{100} \mathrm{Mo}$ nuclei using ${ }^{48 \mathrm{depl}} \mathrm{Ca}^{100} \mathrm{MoO}_{4}$ (CMO) scintillating crystals operating at milliKelvin (mK) temperatures.

\section{AMoRE Experiment}

The AMoRE experiment will run in two phases [4]; the first phase of experiment (AMoRE-I) is going to use a $\sim 5 \mathrm{~kg}$ array of CMO crystals at the Yang Yang underground laboratory (Y2L) in Korea, $\sim 700 \mathrm{~m}$ depth, and the second phase of experiment (AMoRE-II) is going to use $\sim 200 \mathrm{~kg}$ of CMO crystals at a new underground laboratory. Currently, AMoRE-Pilot set-up with $1.8 \mathrm{~kg}$ of CMO crystals is running since 2015 with several upgrades of the detector aiming at achievement of high detector performance and low level of background. The AMoRE-I experiment should start beginning of 2018 in the AMoRE-Pilot measurement configuration with an increased crystal mass.

\subsection{CMO Crystals}

The AMoRE-I experiment is going to use CMO crystal scintillators with calcium depleted in

${ }^{48} \mathrm{Ca}$ and molybdenum enriched in ${ }^{100} \mathrm{Mo}$ as a source of $0 v \beta \beta$ decays. A $Q_{\beta \beta}$-value of ${ }^{100} \mathrm{Mo}$ is $3034 \mathrm{keV}$, which is higher than the most common $\gamma$ background energy, $2.61 \mathrm{MeV}$. Calcium should be depleted in ${ }^{48} \mathrm{Ca}$ since the $2 v \beta \beta$ decay of ${ }^{48} \mathrm{Ca}$ could be potential background. The $\mathrm{CMO}$ crystals have been developed by JSC FOMOS-Materials in Russia and the AMoRE collaborators. Radioactive contamination of the CMO crystals by ${ }^{238} \mathrm{U},{ }^{235} \mathrm{U},{ }^{232} \mathrm{Th}$, and their daughters was measured by a $4 \pi$ gamma measurement system, composed of $14 \mathrm{CsI}$ :Tl scintillating crystals, at the Y2L [5]. In addition, optical and scintillation properties such as the attenuation length, maximum emission wavelength, the absolute light yield of CMO crystals have been measured in a wide temperature interval $7 \mathrm{~K}-300 \mathrm{~K}[6,7,8]$.

\subsection{Detectors for $0 v \beta \beta$ Measurements}

A cryogenic phonon-scintillation detector with metallic magnetic calorimeter (MMC) has been developed [9]. A detector module consists of a CMO crystal, surrounded by reflecting film and supported by copper frame, and MMCs, which are temperature sensors operating at milli-kelvin temperature. Both heat (phonon) and light signals from CMO crystals are measured though Superconducting Quantum Interference Device (SQUID) and MMC sensors. The heat in a crystal is 
measured by MMC and magnetization change in the MMC is readout by SQUID. The simultaneous measurements of phonon and light signals provide clear separation between $\alpha$ and $\beta / \gamma$ events. Detailed principles and designs of detector and sensors are in $[4,10,11,12,13]$. Recent measurements with prototype detectors during engineering operations showed an energy resolution of 8.7 $\mathrm{keV}$ FWHM at $2.6 \mathrm{MeV}$ and clear separations between $\alpha$ and $\beta / \gamma$ events [12].

\subsection{Refrigerator and Shields}

The CMO detector assembly is placed in the cryogen-free dilution refrigerator (CFDR) system, which uses a pulse tube refrigerator, providing sufficient cooling power for the system. Performance of the dilution refrigerator confirmed that base temperature of the system reaches below 10 $\mathrm{mK}$ and that it has $1.6 \mu \mathrm{W}$ of cooling power at $10 \mathrm{mK}$ in normal operating condition [13]. In order to isolate the detector system from vibrations of the cryogenic system, $R \& D$ has been on-going and possible options were considered such as active damper. Recently, a mass spring system has been developed and installed into the CFDR [14].

The dilution refrigerator is surrounded by a 15 -cm-thick low-radioactivity lead shield as shown in Fig. 1 to suppress radioactive backgrounds such as $\gamma$-rays from rocks and other materials. Inside the dilution refrigerator, a 10 -cm-thick low-radioactivity lead plate was placed just above the detector assembly to protect detectors from radioactive backgrounds from materials inside the dilution refrigerator. The detector assembly is surrounded by a $2-\mathrm{mm}$ thick superconducting shield, made of low-radioactivity lead.

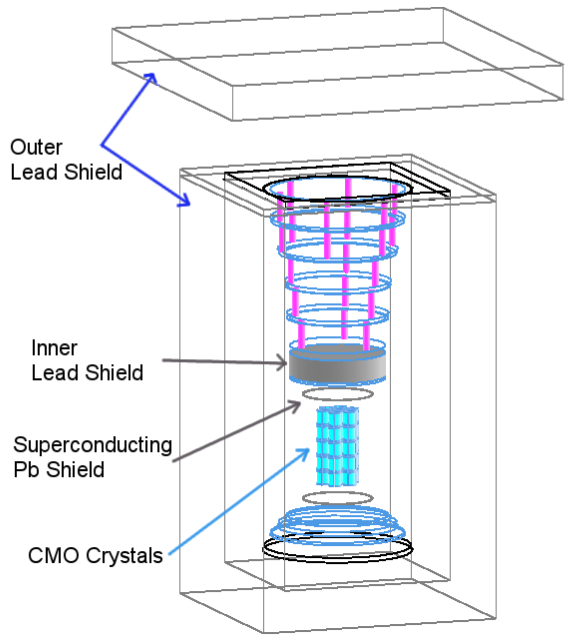

(a)

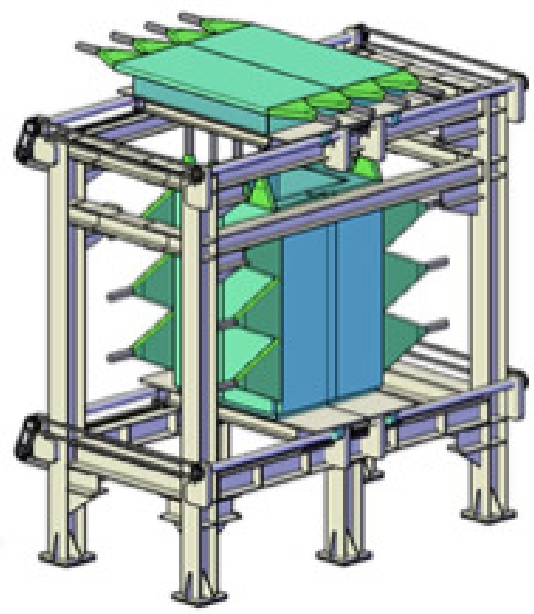

(b)

Figure 1: (a) Schematic view of the refrigerator with CMO detectors array surrounded by passive shield. Top and bottom plates of cylindrical cans of the refrigerator are shown only. (b) The cryostat and the lead shield with muon veto counters inside of the iron structure frame.

\subsection{Muon Veto Counter}

Muon veto counter for AMoRE-I experiment consists of ten 5-cm thick plastic scintillator panels, two panels on the top (each $1725 \times 762 \mathrm{~mm}^{2}$ ) and on all sides of the set-up (four of $1700 \times 500$ 
$\mathrm{mm}^{2}$ and four of $1700 \times 660 \mathrm{~mm}^{2}$ ). Each scintillator panel is viewed by 2-inch Photomultiplier tubes (PMTs) via plastic light guide, as shown in Fig. 1. PMTs were mounted on one side or both sides of scintillator panels depending on accessibility. Signals from the PMTs are digitized and integrated by a $64 \mathrm{MHz}$-sampling Analog-digital-converter (ADC). Before the muon veto system was installed, it has operated for a few months in a stacked-up configuration and it is confirmed that muon signal is clearly separated from $\gamma$ background. Recently, the muon veto system has been installed on sides of the setup.

\subsection{Data Acquisition (DAQ) System}

Readout electronics modules for AMoRE-I experiment consists of three modules, AMoREADC, M64ADC, and Time and Clock Board (TCB). AMoRE-ADC is a $2 \mathrm{MHz}$-sampling ADC with 16 channels for crystal measurements, M64ADC is a $64 \mathrm{MHz}$-sampling ADC with 32 channels for muon veto system, and TCB is a module for synchronizing trigger and clock between crystal and muon veto measurements. The AMoRE-ADC provides two modes, recording data continuously or triggered events, by switching firmware. Data acquisition software was developed on $\mathrm{c}++$ and python programming language in linux environment. The DAQ electronics and software have been tested and operated during the commissioning runs.

\section{Background Simulation}

Background simulation has been performed for the AMoRE-I detector and shield configuration using GEANT4 simulation toolkit [15]. Background sources such as ${ }^{238} \mathrm{U},{ }^{232} \mathrm{Th},{ }^{235} \mathrm{U}$, and ${ }^{210} \mathrm{~Pb}$ inside the crystals, reflector, copper frame, surrounding materials, outer and inner lead shields, and rock walls of the Y2L cavity were simulated and effects on CMO crystals were estimated based on measured and reported radioactive contaminations of the materials. The estimated background rate in the region of interest $(3.034 \pm 0.01 \mathrm{MeV})$ for AMoRE-I experiment configuration was estimated to be $<1.5 \times 10^{-3}$ counts $/ \mathrm{keV} / \mathrm{kg} / \mathrm{yr}$ [16]. The effects of random coincidences between backgrounds and $2 v \beta \beta$ decay of ${ }^{100} \mathrm{Mo}$, including two $2 v \beta \beta$ decays of ${ }^{100} \mathrm{Mo}$, were estimated to be $<2.3 \times 10^{-4}$ counts $/ \mathrm{keV} / \mathrm{kg} / \mathrm{yr}$. The total expected background rate, $<1.7 \times 10^{-3}$ counts $/ \mathrm{keV} / \mathrm{kg} / \mathrm{yr}$, was lower than a goal of AMoRE-I background limit for zero background condition, $2 \times 10^{-3}$ counts $/ \mathrm{keV} / \mathrm{kg} / \mathrm{yr}$.

As shown in Fig. 2, an internal background from crystals are the most dominant and materials near crystals are the second dominant source. Further background simulations with a realistic detector design and materials of AMoRE-Pilot configuration are ongoing.

\section{Current Status and Plans}

AMoRE-Pilot commissioning runs have started with five crystals $(\sim 1.5 \mathrm{~kg})$ since Sep. 2015. During commission runs, crystal internal background measurements, system noise R\&D, detector $\mathrm{R} \& \mathrm{D}$, data acquisition system debugging, and trigger algorithm development have been carried out. After debugging and upgrading the system, recently, the fifth run with six crystals $(\sim 1.8 \mathrm{~kg})$ has started. Online monitoring system was implemented. Detector status and DAQ status are recorded into database and status and plots are available on the web, as shown in Fig. 3. We are planning 


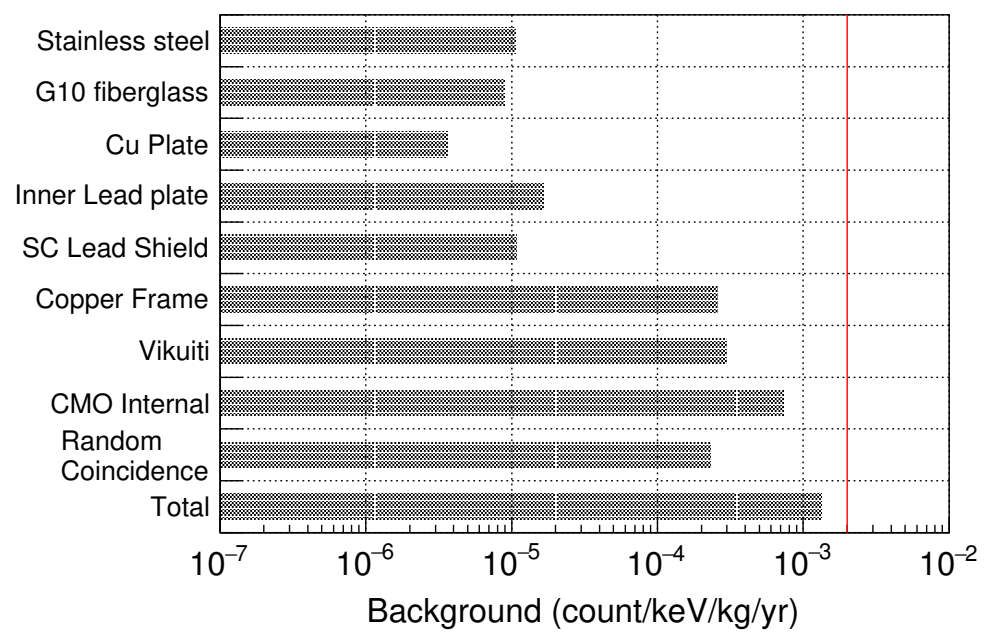

Figure 2: Background budgets in the ROI from the cryostat and materials.

to operate the AMoRE-Pilot phase by the end of this year and detector upgrades to AMoRE-I experiment is planned in next year. Assuming 5-year measurements with $5 \mathrm{~kg}$ of CMO crystals, it is expected a half-life sensitivity $T_{1 / 2}^{0 v} \sim 10^{25}$ years at $90 \%$ C.L. in zero-backgroud condition.

The second phase of AMoRE (AMoRE-II) experiment with $200 \mathrm{~kg}$ molybdate crystal scintillators is going to be operating at a new underground laboratory in Korea, which will be a deeper place than Y2L, in a few years. For AMoRE-II, laboratory construction as well as R\&Ds such as powder purification, $R \& D$ of molybdenum containing crystal scintillators, crystal growing, detector design, shielding study, background study using simulation are ongoing.

\section{Acknowledgments}

We thank the Korea Hydro and Nuclear Power (KHNP) Company for providing laboratory space and supports. This research is funded by the Institute for Basic Science, Korea under project code IBS-R016-D1.

\section{References}

[1] Particle Data Group collaboration, C. Patrignani et al., Review of Particle Physics, Chin. Phys. C40 (2016) 100001.

[2] C. Giunti and C. W. Kim, Fundamentals of Neutrino Physics and Astrophysics. Oxford, UK: Univ. Pr. (2007) 710 p.

[3] H. Bhang et al., AMoRE experiment: a search for neutrinoless double beta decay of ${ }^{100}$ Mo isotope with ${ }^{40} \mathrm{Ca}^{100} \mathrm{MoO}_{4}$ cryogenic scintillation detector, J. Phys. Conf. Ser. 375 (2012) 042023.

[4] AMoRE collaboration, V. Alenkov et al., Technical Design Report for the AMoRE 0v $\beta \beta$ Decay Search Experiment, Arxiv 1512.05957. 




Figure 3: Screenshot of DAQ status online monitoring page.

[5] J. Y. Lee et al., A Study of Radioactive Contamination of ${ }^{40} \mathrm{Ca}^{100} \mathrm{MoO}_{4}$ Crystals for the AMoRE Experiment, IEEE Trans. Nucl. Sci. 63 (2016) 543-547.

[6] V. B. Mikhailik, S. Henry, H. Kraus and I. Solsky, Temperature dependence of CaMoO $\mathrm{Scintillation}$ properties, Nucl. Instrum. Meth. A583 (2007) 350-355.

[7] H. Kim et al., Neutrino-Less Double Beta Decay Experiment Using $\mathrm{Ca}^{100} \mathrm{MoO}_{4}$ Scintillation Crystals, IEEE Trans. Nucl. Sci. 57 (2010) 1475-1480.

[8] J. H. So et al., Scintillation Properties and Internal Background Study of ${ }^{40} \mathrm{Ca}^{100} \mathrm{MoO}_{4} \mathrm{Crystal}$ Scintillators for Neutrino-Less Double Beta Decay Search, IEEE Trans. Nucl. Sci. 59 (2012) 2214-2218.

[9] W. S. Yoon, C. S. Kang, S. R. Kim, G. B. Kim, H. J. Lee, M. K. Lee et al., Development of a high resolution alpha spectrometer using a magnetic calorimeter, Nucl. Instrum. Meth. A784 (2015) 143-146.

[10] H. J. Lee, J. H. So, C. S. Kang, G. B. Kim, S. R. Kim, J. H. Lee et al., Development of a scintillation light detector for a cryogenic rare-event-search experiment, Nucl. Instrum. Meth. A784 (2015) $508-512$.

[11] G. B. Kim et al., Heat and Light Measurement of ${ }^{40} \mathrm{Ca}^{100} \mathrm{MoO}_{4}$ Crystal for the AMoRE Double Beta Decay Experiment, IEEE Trans. Nucl. Sci. 63 (2016) 539-542.

[12] G. B. Kim et al., Novel measurement method of heat and light detection for neutrinoless double beta decay, Astropart. Phys. 91 (2017) 105-112.

[13] C. Kang et al., MMC-based low-temperature detector system of the AMoRE-Pilot experiment, Superconductor Science and Technology Accepted. 
[14] C. Lee, H. S. Jo, C. S. Kang, G. B. Kim, I. Kim, S. R. Kim et al., Vibration isolation system for cryogenic phonon-scintillation calorimeters, JINST 12 (2017) C02057.

[15] Geant4 collaboration, M. Asai, A. Dotti, M. Verderi and D. H. Wright, Recent developments in Geant4, Annals Nucl. Energy 82 (2015) 19-28.

[16] A. Luqman et al., Simulations of background sources in AMoRE-I experiment, Nucl. Instrum. Meth. A855 (2017) 140-147, [1601.01249]. 